Article

\title{
Ferronematics Based on Paramagnetic Nitroxide Radical Liquid Crystal
}

\author{
Takuya Akita ${ }^{1}$, Yoshiaki Uchida ${ }^{1,2, *}$, Sho Nakagami ${ }^{1}$, Daichi Kiyohara ${ }^{1}$ and \\ Norikazu Nishiyama ${ }^{1}$
}

1 Graduate School of Engineering Science, Osaka University, 1-3 Machikaneyama-cho, Toyonaka, Osaka 560-8531, Japan; E-Mails: t.akita@cheng.es.osaka-u.ac.jp (T.A.); u010445c@gmail.com (S.N.); kiyo629518@gmail.com (D.K.); nisiyama@cheng.es.osaka-u.ac.jp (N.N.)

2 PRESTO, Japan Science and Technology Agency (JST), 4-1-8 Honcho, Kawaguchi, Saitama 332-0012, Japan

* Author to whom correspondence should be addressed; E-Mail: yuchida@cheng.es.osaka-u.ac.jp; Tel./Fax: +81-6-6850-6256.

Academic Editor: Hiroyuki Yoshida

Received: 23 March 2015 / Accepted: 20 April 2015 / Published: 27 April 2015

\begin{abstract}
We have prepared novel ferronematics based on a paramagnetic liquid crystalline (LC) material. Our ferronematics can disperse a higher volume fraction of magnetic nanoparticles compared to classical ferronematics because paramagnetic nature of the host LC material prevents the aggregation of magnetic nanoparticles. The interactions between the magnetic nanoparticles and the LC material enhance a magnetic anisotropy of ferronematics and improve the magnetic responsivity.
\end{abstract}

Keywords: ferronematics; paramagnetic liquid crystals; $\mathrm{Fe}_{3} \mathrm{O}_{4}$ nanoparticles; Frederiks threshold; magnetic-field-induced molecular reorientation

\section{Introduction}

The orientational direction of liquid crystalline (LC) phases can be controlled by applying an external electric or magnetic field because of their anisotropic dielectric permittivity or magnetic susceptibility [1]. Since a strong magnetic field $(\boldsymbol{B} \sim 1 \mathrm{~T})$ is required to realize the magnetic-field-induced 
molecular reorientation, in practice most LC devices such as displays are driven by an electric field. This is because most LC materials have a small anisotropy of magnetic susceptibility $\left(\Delta \chi \sim 10^{-7}\right)[2]$. However, the magnetic-field-induced molecular reorientation should be suitable for devices consisting of LC materials like ionic LC materials, to which the electric-field-induced molecular reorientation is not applicable [3]. In actual use, the responsivity of LC materials to a magnetic field needs to be improved in some way.

In 1970, Brochard and de Gennes theoretically proposed the way to improve the magnetic responsivity of LC materials [4]. They demonstrated that LC materials doped with magnetic particles at very low volume fractions could exhibit high responsivity to applied magnetic field; the composite systems are known as "ferronematics". The high responsivity induced by an orientational coupling between the magnetic particles and the LC director field gives possibilities of the magnetic field-driven LC devices. In 1983, Chen and Amer experimentally succeeded in the reorientation of ferronematics by a few milli-tesla of magnetic field [5]. A number of ferronematics have been reported [6,7]; they exhibit the high magnetic responsivity [8] and unusual magnetic behaviors such as linear responses of birefringence [9] and capacitance [10] in a low magnetic field region (far below the Frederiks threshold).

In contrast to the previously reported ferronematics consisting of diamagnetic LC materials as the hosts, we see a great future for some ferronematics with paramagnetic liquid crystalline (PLC) hosts. This is because PLC hosts are expected to work as dispersion media for magnetic particles better than diamagnetic LC hosts and to exhibit the coupling between the magnetic moments of magnetic particles and paramagnetic susceptibility of PLC molecules. They are classified into two categories; the majority is metal-complex LC compounds containing paramagnetic transition or lanthanide metal ions [11,12], and the minority is all-organic radical LC compounds. Despite the existence of large magnetic anisotropy of the former metal-complexes, their high viscosity derived from the polar character frequently renders the magnetic-field-induced reorientation difficult. In contrast, the latter all-organic radical LC materials with the small magnetic anisotropy do not show such high viscosity. As one of the most stable all-organic radical PLC materials, a nitroxide radical LC compound $\mathbf{1}$ with an enantiotropic nematic phase has been reported to exhibit the magnetic-field-induced molecular reorientation in the nematic phase at below $1 \mathrm{~T}$ of magnetic field $[3,13]$. If the magnetic responsivity is improved by doping magnetic particles, the all-organic radical LC materials would be more suitable for the hosts of ferronematics. Here, we demonstrate the preparation of the ferronematics consisting of $\mathrm{Fe}_{3} \mathrm{O}_{4}$ magnetic nanoparticles (MNPs) to the nematic phase of $\mathbf{1}$ and discuss the MNPs dispersibility and the magnetic-field-induced Frederiks transition of the new ferronematics.

\section{Experimental}

The studied ferronematics were based on the PLC material 1, which was prepared as the previous reported procedure [14]. The chemical structure and phase transition temperatures of the thermotropic nematic compound $\mathbf{1}$ are shown in Figure 1. In spite of the existence of a radical moiety, $\mathbf{1}$ is thermally stable up to about $150{ }^{\circ} \mathrm{C}$ in air. The ferronematics were simply prepared by adding a toluene solution of $\mathrm{Fe}_{3} \mathrm{O}_{4} \mathrm{MNPs}$ coated with oleic acid (Sigma Aldrich, St. Louis, MO, USA) to 1. Then, the mixture was left at the temperature of $\sim 120{ }^{\circ} \mathrm{C}$ so that the solvent could evaporate and $\mathbf{1}$ would show an isotropic phase. The size and morphology of the MNPs were determined by TEM. They were almost spheres of 
mean diameter of $\sim 20 \mathrm{~nm}$. The MNPs in the prepared ferronematics have volume fractions $\phi=0,1 \times 10^{-5}, 1 \times 10^{-4}, 1 \times 10^{-3}$, which are named $\mathbf{1}, \mathbf{2 a}, \mathbf{2 b}$ and $\mathbf{2 c}$, respectively. Phase transition temperatures of each sample were determined by differential scanning calorimetry (DSC) (SHIMADZU DSC-60) at a scan rate of $2{ }^{\circ} \mathrm{C} / \mathrm{min}$.

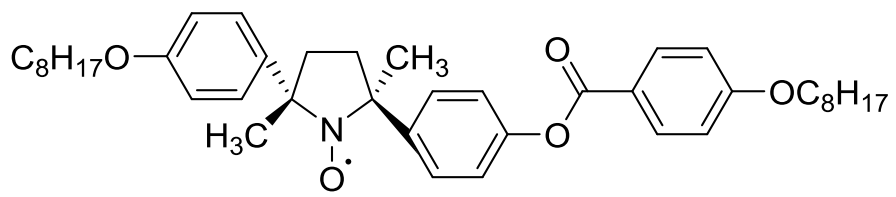

1: $\operatorname{Cr} 73.7 \mathrm{~N} 104.0$ Iso

Figure 1. Chemical structure of one of the enantiomers of $\mathbf{1}(2 S, 5 S)$ and phase transition temperatures $\left({ }^{\circ} \mathrm{C}\right)$ of racemic 1 . The temperatures were determined by differential scanning calorimetry (DSC) upon heating. Standard notation gives the transition temperatures between the crystalline $(\mathrm{Cr})$, nematic $(\mathrm{N})$ and isotropic (Iso) phases.

The Frederiks thresholds were measured for $\mathbf{1}$ and $\mathbf{2 a - c}$ by a polarization-optics method. The ferronematics were introduced by capillary action into about $10 \mu \mathrm{m}$ thick $(D)$ handmade sandwich cells $(7.5 \mathrm{~mm} \times 10 \mathrm{~mm})$ in which the inner surfaces of the two glass substrates were coated with polyimide (AL1254, JSR, Tokyo, Japan). The polyimide surfaces in the cell were rubbed horizontally 10 times using a velvet roller before bonded together. The magneto-optical measurements were performed at the temperature of $80{ }^{\circ} \mathrm{C}$ (far below the magnetic transition temperature of MNPs [15]) with our experimental setup shown in Figure 2. The easy axis of molecular direction $\boldsymbol{n}$ and the analyzer direction were parallel to each other, and a magnetic field $\boldsymbol{B}$ perpendicular to $\boldsymbol{n}$ and parallel to the cell plane was applied. To discuss the molecular reorientation induced by the magnetic field $\boldsymbol{B}$, we monitored the intensity of transmitted light of laser beam with wavelength of $532 \mathrm{~nm}$.

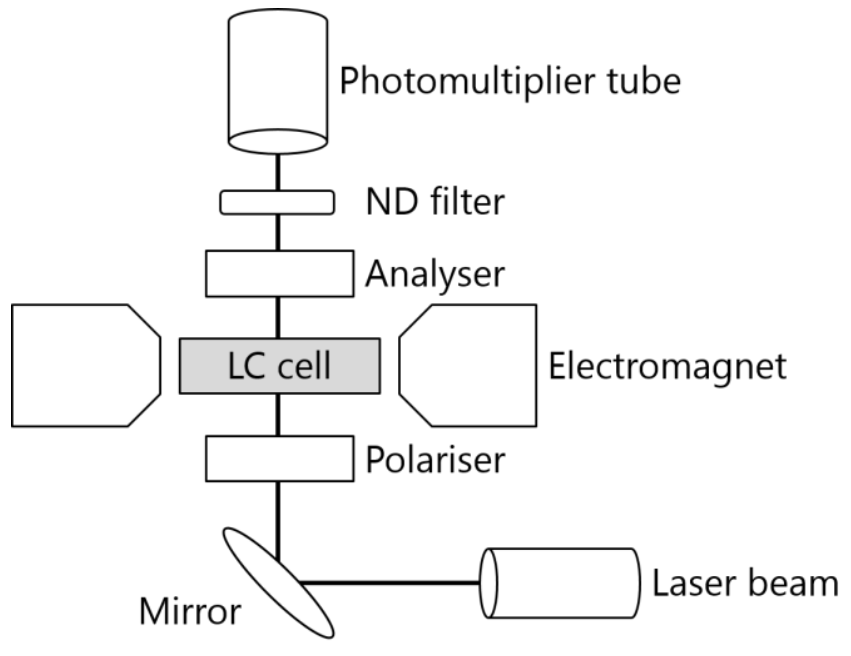

(a)

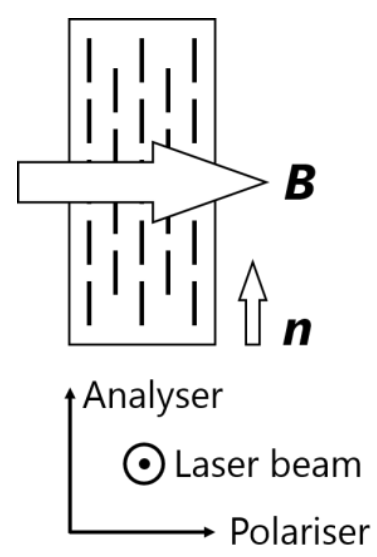

(b)

Figure 2. (a) Experimental setup for magneto-optical measurements; (b) Top view of the liquid crystalline (LC) cell in the initial state. 


\section{Results and Discussion}

We carried out simple tests of the dispersibility of MNPs into LC matrices with the use of optical transmission microscopy at the temperature where samples exhibits the isotropic phase. We compared the size of aggregates in PLC material 1 with that in a conventional diamagnetic LC material 4-cyano-4'-heptyloxybiphenyl (7OCB) doped with the same MNPs $\left(\phi=1 \times 10^{-3}\right)$. A lot of large aggregates were observed in 7OCB as shown in Figure 3a. In contrast, such phenomena were suppressed in our ferronematics based on $\mathbf{1}$ as shown in Figure 3b. The aggregation of MNPs tends to be promoted in LC phases and they do not disperse again even if the temperature is elevated up to $\sim 120{ }^{\circ} \mathrm{C}$ where they exhibit isotropic phase. This difference of the dispersibility can be attributed to the magnetism of each LC material. On the one hand, diamagnetic LC molecules are repelled by MNPs; on the other hand, PLC molecules are attracted by the same MNPs. In fact, the droplets made of PLC material 1 floating on hot water are attracted by a magnet [16]. Therefore, PLC material $\mathbf{1}$ is desirable as a host material of ferronematics.

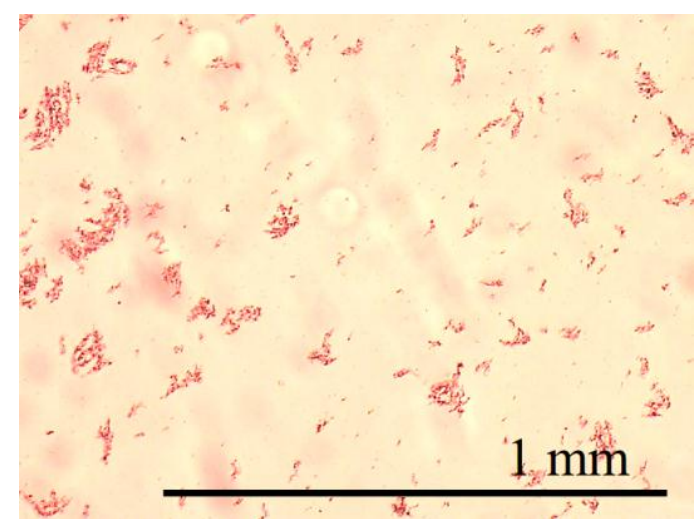

(a)

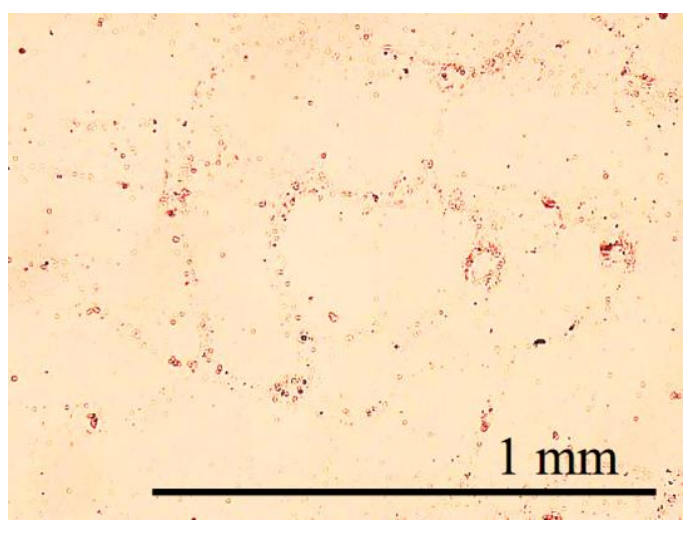

(b)

Figure 3. Bright-field micrographs of ferronematics based on (a) 4-cyano-4'-heptyloxybiphenyl (7OCB) and (b) paramagnetic liquid crystalline (PLC) material 1 at $120{ }^{\circ} \mathrm{C}$ where they exhibit isotropic phase $\left(\phi=1 \times 10^{-3}\right)$.

In a certain diamagnetic nematic material MLC-6609 (Merck, Darmstadt, Germany) doped with ferroelectric $\mathrm{BaTiO}_{3}$ nanoparticles, the nanoparticles induce the increase of the orientational order parameter resulting in $9^{\circ} \mathrm{C}$ rise of nematic-isotropic phase transition temperature, $T_{\mathrm{NI}}[17,18]$. Since the similar effect might occur for our ferronematics due to the magnetic moments of MNPs and paramagnetic susceptibility of the PLC molecules, we measured $T_{\mathrm{NIS}}$ of the samples $\mathbf{1}$ and $\mathbf{2 a}-\mathbf{c}$ by DSC analysis. As a result, the more concentrated the MNPs become, the lower the phase transition temperature becomes as shown in Table 1. Therefore, above-mentioned increase of orientational order leading to the rise of $T_{\mathrm{NI}}$ was not observed in our ferronematics. Thus, these results suggest that the orientational order is not induced by MNPs or the effect is too small to observe and the impurity effect of the MNPs to disturb the orientational order is dominant. In particular, the depression of $T_{\mathrm{NI}}$ obviously appears in 2c with the highest volume fraction of MNPs. This result indicates that the molecular alignment of $\mathbf{2 c}$ is somewhat disturbed by the doped MNPs especially nearby $T_{\mathrm{NI}}$. 
Table 1. Nematic-isotropic phase transition temperatures of $\mathbf{1}$ and $\mathbf{2 a}-\mathbf{c}$.

\begin{tabular}{ccc}
\hline Sample & $\boldsymbol{\Phi}[-]$ & $\boldsymbol{T}_{\mathrm{NI}}\left[{ }^{\circ} \mathbf{C}\right]$ \\
\hline $\mathbf{1}$ & 0 & 104.0 \\
$\mathbf{2 a}$ & $1 \times 10^{-5}$ & 102.7 \\
$\mathbf{2 b}$ & $1 \times 10^{-4}$ & 101.2 \\
$\mathbf{2 c}$ & $1 \times 10^{-3}$ & 92.6 \\
\hline
\end{tabular}

Molecules in the LC cell started to align along $\boldsymbol{B}$ as shown in Figure 4 at a certain critical value of the magnetic field, Frederiks threshold $B_{\mathrm{C}}$, which can be described for undoped nematic LC cell as [2]:

$$
B_{\mathrm{C}}=\frac{\pi}{D} \sqrt{\frac{\mu_{0} K_{2}}{\Delta \chi}}
$$

where $\mu_{0}$ is magnetic permeability and $K_{2}$ is LC twist elastic constant. The magnetic-field-induced molecular reorientation of LC materials is mainly caused by $\Delta \chi$, which is the sum of the paramagnetic component $\left(\Delta \chi_{\text {para }}\right)$ and the diamagnetic one $\left(\Delta \chi_{\text {dia }}\right)$ for PLC materials unlike the conventional diamagnetic LC materials. This twist deformation of molecular orientation caused an increase of transmitted light intensity under crossed nicols. The relative intensity $I / I_{0}$ for the samples $\mathbf{1}$ and $\mathbf{2 a - c}$ is plotted as a function of the magnetic field $\boldsymbol{B}$ in Figure 5, where $I_{0}$ is transmitted light intensity measured under parallel nicols. For the undoped sample 1, the molecular reorientation started at about 0.8 T. Due to doping MNPs, magnetic responsivity of the PLC material was enhanced; the Frederiks threshold decreased, or the larger orientational deformation was induced at the same magnetic field. These ferronematics showed the same order of magnitude of the magnetic responsivity compared to the classical ferronematics. It is because $\Delta \chi_{\text {para }}$ hardly affects the magnetic responsivity because $\Delta \chi_{\text {dia }} \gg \Delta \chi_{\text {para }}\left(\Delta \chi_{\text {dia }}=6.5 \times 10^{-5} \mathrm{emu} \cdot \mathrm{mol}^{-1}\right.$ and $\Delta \chi_{\text {para }}=-1.7 \times 10^{-6} \mathrm{emu} \cdot \mathrm{mol}^{-1}$ at $300 \mathrm{~K}$ [3] $)$.

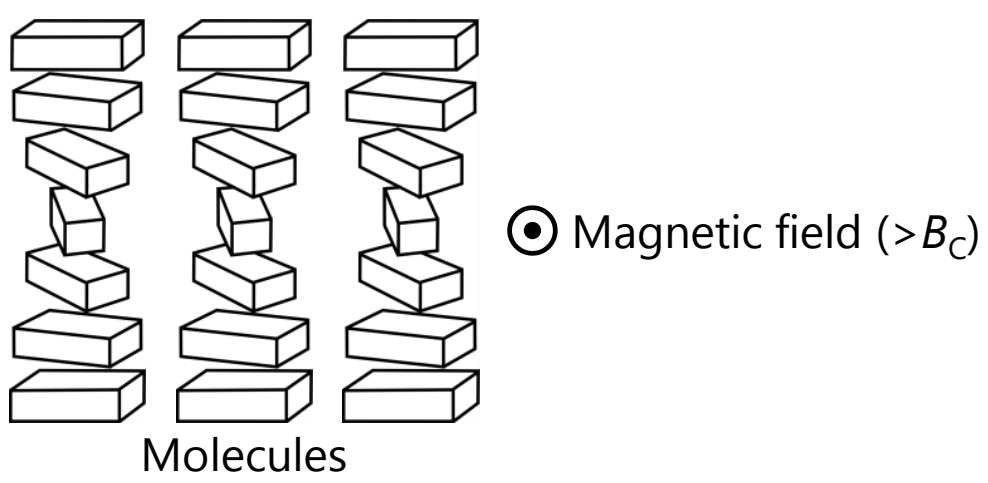

Figure 4. Schematic illustration of twist deformation of molecular alignments induced by applied magnetic field. Molecules in closer to the center reorient along to magnetic field. 


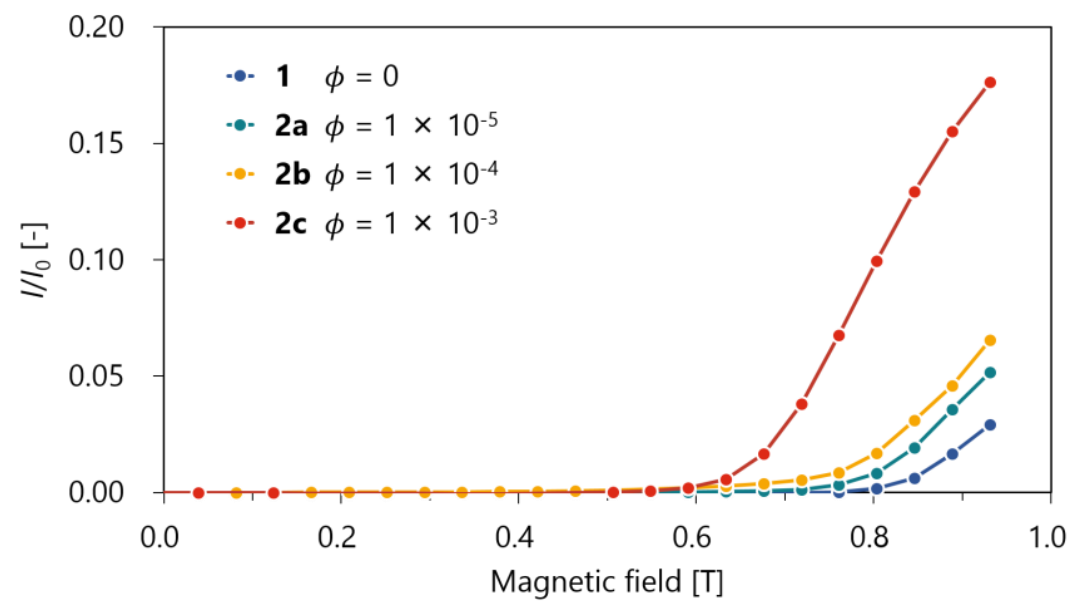

Figure 5. Relative intensity $\left(I / I_{0}\right)$ as a function of the applied magnetic field $(B)$, for $\mathbf{1}$ and $2 \mathbf{a}-\mathbf{c}$.

The free energy density for the ferronematics with respect to the elastic deformation is written as $[19,20]$ :

$$
\begin{aligned}
F=\frac{1}{2}\left[K_{1}(\boldsymbol{\nabla} \cdot \boldsymbol{n})^{2}\right. & \left.+K_{2}(\boldsymbol{n} \cdot \boldsymbol{\nabla} \times \boldsymbol{n})^{2}+K_{3}(\boldsymbol{n} \times \boldsymbol{\nabla} \times \boldsymbol{n})^{2}\right] \\
& -\frac{1}{2} \Delta \chi(\boldsymbol{n} \cdot \boldsymbol{H})^{2}+\frac{\phi k_{\mathrm{B}} T}{v} \ln \phi-\phi M_{\mathrm{S}}(\boldsymbol{m} \cdot \boldsymbol{H})+\phi W(\boldsymbol{n} \cdot \boldsymbol{m})^{2}
\end{aligned}
$$

where $\boldsymbol{n}$ is the nematic director, $v$ is the particle volume, $\boldsymbol{m}$ is the magnetic director of MNP, $K_{1}$ and $K_{3}$ are the LC splay and bend elastic constants, $M_{\mathrm{S}}$ is the MNP magnetization and $W$ is the coupling energy density between the MNP and the LC orientation. Equation (2) represents the nematic director field interacts with the magnetic moments of MNPs under applied magnetic field. The first three terms represent the usual Frank energy density of the elastic deformations of the nematic director field, the fourth term is the magnetic energy of the nematic director field. The last three terms are specific to ferronematics: the contribution of the mixing entropy of their ideal solution, the magnetic energy of the MNPs and the coupling energy between the MNPs and the nematic director. Since the measurements were carried out at the same temperature far below the $T_{\mathrm{NIS}}$, the change of orientational scalar order parameter $S$ induced by doping MNPs and temperature dependence on interactions between MNPs and LC host are ignorable. Since the effects of the last three terms of Equation (2) derived from the doping MNPs influence the Frederiks threshold $B_{C}$ of Equation (1), they are combined into $\Delta \chi$ causing the Frederiks transition as:

$$
\Delta \chi \rightarrow(1+\theta) \Delta \chi
$$

where $\theta$ only contains the magnetic effects derived from doping MNPs. Namely, the coupling between MNPs and nematic director induced by the applied magnetic field increases $\theta$ and enhances the effective magnetic anisotropy. Then, adding the contribution of $\theta$ into Equation (1), Frederiks threshold decreases. Assuming that $D$ and $K_{2}$ are constant, $\theta(\phi)$ is derived from Equations (1) and (3) as:

$$
\theta(\phi)=\frac{B_{\mathrm{C}}(0)^{2}}{B_{\mathrm{C}}(\phi)^{2}}-1
$$


where $B_{\mathrm{C}}(\phi)$ is defined as the magnetic field when we observed $I / I_{0}=0.01$. Using Equation (4), we calculated $\theta(\phi)$ from $B_{\mathrm{C}}(0)$ and $B_{\mathrm{C}}(\phi)$. As shown in Figure 6 , the interactions between the MNPs and the nematic director increase as a function of $\phi$. In a small $\phi$ region, MNPs doping effects are drastically large. The addition of MNPs into the PLC material results in 1.8-fold enhancement of the magnetic responsivity of the PLC material.

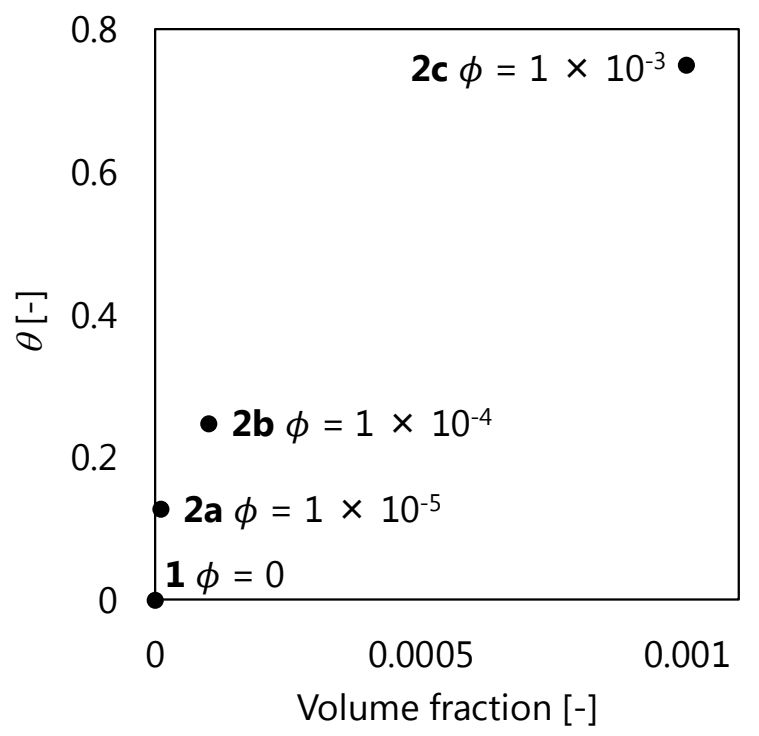

Figure 6. Relationships between volume fraction of magnetic nanoparticles (MNPs) and $\theta$.

\section{Conclusions}

We have fabricated novel ferronematics based on the PLC material 1. The aggregation of MNPs is reduced by the paramagnetic nature of $\mathbf{1}$. Similar to classical ferronematics based on the diamagnetic LC materials, the response to applied magnetic field can be enhanced by doping MNPs. MNPs interact with the nematic orientational order and increase the magnetic anisotropy $\Delta \chi$ of PLC material, which causes the decrease of the Frederiks threshold. Furthermore, if the paramagnetic anisotropy $\Delta \chi_{\text {para }}$ is increased or the LC elastic constants are decreased by molecular modification and more anisotropically shaped MNPs are doped into PLC hosts with larger paramagnetic susceptibility $\chi_{\text {para, }}$ the ferronematics based on PLC materials would be more useful than the conventional ferronematics based on a diamagnetic one.

\section{Acknowledgments}

The authors thank Jun Yamamoto, Kyoto University, for helpful advice. The authors are very grateful to JSR Corporation for providing AL1254. This work was supported in part by the Japan Science and Technology Agency (JST) "Precursory Research for Embryonic Science and Technology (PRESTO)" for a project of "Molecular technology and creation of new function", by ICHJYU Industrial Science and Technology Promotion Foundation and by Foundation for Promotion of Izumi Science and Technology Foundation. 


\section{Author Contributions}

Takuya Akita carried out most of the sample measurements and analysis, and wrote the manuscript. Yoshiaki Uchida designed the study and helped to analyze the data. Sho Nakagami and Daichi Kiyohara prepared the LC samples. Norikazu Nishiyama supervised the project.

\section{Conflicts of Interest}

The authors declare no conflict of interest.

\section{References}

1. Frederiks, V.; Zolina, V. Forces causing the orientation of an anisotropic liquid. Trans. Faraday Soc. 1933, 29, 919-930.

2. De Gennes, P.G.; Prost, J. The Physics of Liquid Crystals, 2nd ed.; Clarendon Press: Oxford, UK, 1993.

3. Uchida, Y.; Tamura, R.; Ikuma, N.; Shimono, S.; Yamauchi, J.; Shimbo, Y.; Takezoe, H.; Aoki, Y.; Nohira, H. Magnetic-field-induced molecular alignment in an achiral liquid crystal spin-labeled by a nitroxyl group in the mesogen core. J. Mater. Chem. 2009, 19, 415-418.

4. Brochard, F.; de Gennes, P.G. Theory of magnetic suspensions in liquid crystals. J. Phys. 1970, 31, 691-708.

5. Chen, S.-H.; Amer, N.M. Observation of macroscopic collective behavior and new texture in magnetically doped liquid crystals. Phys. Rev. Lett. 1983, 51, 2298-2301.

6. Tomašovièová, N.; Kopèanský, P.; Éber, N. Magnetically Active Anisotropic Fluids Based on Liquid Crystals, in Anisotropy Research: New Developments; Lemu, H., Ed.; Nova Science Publishers: Hauppauge, NY, USA, 2012; pp. 245-276.

7. Garbovskiy, Y.; Glushchenko, A. Liquid Crystalline Colloids of Nanoparticles: Preparation, Properties, and Applications. Solid State Phys. 2010, 62, 1-74.

8. Garbovskiy, Y.; Baptist, J.R.; Thompson, J.; Hunter, T.; Lim, J.H.; Gi Min, S.; Wiley, J.B.; Malkinski, L.M.; Glushchenko, A.; Celinski, Z.; et al. Increasing the switching speed of liquid crystal devices with magnetic nanorods. Appl. Phys. Lett. 2012, 101, 181109:1-181109:5.

9. Podoliak, N.; Buchnev, O.; Buluy, O.; D’Alessandro, G.; Kaczmarek, M.; Reznikov, Y.; Sluckin, T.J. Macroscopic optical effects in low concentration ferronematics. Soft Matter 2011, 7 , 4742-4749.

10. Tomašovičová, N.; Timko, M.; Mitróová, Z.; Koneracká, M.; Rajňak, M.; Éber, N.; Tóth-Katona, T.; Chaud, X.; Jadzyn, J.; Kopčanský, P.; et al. Capacitance changes in ferronematic liquid crystals induced by low magnetic fields. Phys. Rev. E 2013, 87, 014501.

11. Griesar, K.; Galyametdinov, Y.; Athanassopoulou, M.; Ovchinnikov, I.; Haase, W. Paramagnetic liquid crystalline nickel (II) compounds. Adv. Mater. 1994, 6, 381-384.

12. Binnemans, K.; Galyametdinov, Y.G.; van Deun, R.; Bruce, D.W.; Collinson, S.R.; Polishchuk, A.P.; Bikchantaev, I.; Haase, W.; Prosvirin, A.V.; Tinchurina, L.; et al. Rare-Earth-Containing Magnetic Liquid Crystals. J. Am. Chem. Soc. 2000, 122, 4335-4344. 
13. Ikuma, N.; Tamura, R.; Shimono, S.; Kawame, N.; Tamada, O.; Sakai, N.; Yamauchi, J.; Yamamoto, Y. Magnetic properties of all-organic liquid crystals containing a chiral five-membered cyclic nitroxide unit within the rigid core. Angew. Chem. Int. Ed. Engl. 2004, 43, 3677-3682.

14. Ikuma, N.; Tamura, R.; Masaki, K.; Uchida, Y.; Shimono, S.; Yamauchi, J.; Aoki, Y.; Nohira, H. Paramagnetic FLCs Containing an Organic Radical Component. Ferroelectrics 2006, 343, 119-125.

15. Thapa, D.; Palkar, V.R.; Kurup, M.B.; Malik, S.K. Properties of magnetite nanoparticles synthesized through a novel chemical route. Mater. Lett. 2004, 58, 2692-2694.

16. Uchida, Y.; Suzuki, K.; Tamura, R.; Ikuma, N.; Shimono, S.; Noda, Y.; Yamauchi, J. Anisotropic and inhomogeneous magnetic interactions observed in all-organic nitroxide radical liquid crystals. J. Am. Chem. Soc. 2010, 132, 9746-9752.

17. Li, F.; Buchnev, O.; Cheon, C.; Glushchenko, A.; Reshetnyak, V.; Reznikov, Y.; Sluckin, T.; West, J. Orientational Coupling Amplification in Ferroelectric Nematic Colloids. Phys. Rev. Lett. 2006, 97, 147801:1-147801:4.

18. Li, F.; Buchnev, O.; Cheon, C.; Glushchenko, A.; Reshetnyak, V.; Reznikov, Y.; Sluckin, T.; West, J. Erratum: Orientational Coupling Amplification in Ferroelectric Nematic Colloids [Phys. Rev. Lett. 97, 147801(2006)]. Phys. Rev. Lett. 2007, 99, 219901.

19. Burylov, S.V.; Raikher, Y.L. Magnetic Fredericksz transition in a ferronematic. J. Magn. Magn. Mater. 1993, 122, 62-65.

20. Zadorozhnyi, V.I.; Sluckin, T.J.; Reshetnyak, V.Y.; Thomas, K.S. The Frederiks effect and related phenomena in ferronematic materials. SIAM J. Appl. Math. 2008, 68, 1688-1716.

(C) 2015 by the authors; licensee MDPI, Basel, Switzerland. This article is an open access article distributed under the terms and conditions of the Creative Commons Attribution license (http://creativecommons.org/licenses/by/4.0/). 